Bulletin of the Section of Logic

Volume 46:1/2 (2017), pp. 21-32

http://dx.doi.org/10.18778/0138-0680.46.1.2.03

Jan Woleński

\title{
UNIVERSALITY OF LOGIC
}

\begin{abstract}
This paper deals with the problem of universality property of logic. At first, this property is analyzed in the context of first-order logic. Three senses of the universality property are distinguished: universal applicability, topical neutrality and validity (truth in all models). All theses senses can be proved to be justified. The fourth understanding, namely the amount of expressive power, is connected with the criticism of the first-order thesis: first-order logic is the logic. The categorical approach to logic is presented as associated with the last understanding of universality. The author concludes that two senses of universality should be sharply discriminated and defends the first-order thesis.

Keywords: universality, logica docents, logica utens, first-order logic, consequence operation, model, syntax, semantics, expressive power.

Since the present paper is related to [17], I will briefly recapitulate this earlier contribution with some additions (in fact, I skip most bibliographical references in the summary). The following statement by Petrus Hispanus:

dialectica (that is, logic) est art atrium et scientia scientiarum ad omnium aliarum scientiarum methodorum principia viam habent,

is a good starting point for my further considerations. The idea that logic provides methodological principles for all other sciences, can be taken as expressing the idea that logic is universal. However, the quoted formulation appears to be too vague. Above all, the scope of logic traditionally covers a great variety of topics. Logic in the broad sense includes semiotics, formal logic and methodology of science as its parts. On the other hand, logica
\end{abstract}


sensu stricto is basically identified with formal logic understood as the investigations of principles of deductive reasoning. More formally, formal logic deals with the relation expressed by the sign $\vdash$ (is logically provable) or the consequence operation $C n$ (I will use both symbols in my further considerations.

Yet not everything is clear in the explanation that logic deals with deductive inferences. Firstly, we can think of logic as codifying the socalled logical laws and rules (I omit the question whether logic states or discovers them), for instance, by proposing axiomatizations or other ordering means, for instance, natural deduction formulation. Secondly, logic can be regarded as investigating the properties of logical theorems (rules, systems, etc.) by using proof-theory, model theory and recursion theory. Consequently, logical research includes the work carried out in the objectlanguage as well as in the metalanguage. It is convenient to speak about logic and metalogic (metamathematics), respectively. The distinction of logica docens (theoretical logic) and logica utens (applied logic) has also a considerable merit for understanding what is logic. Whereas the former deals with logical systems and their properties (the label 'pure logic' is sometimes used in this context), the latter concerns logic as a body of principles applied to other fields, particularly mathematics. Since logica utens principally functions as an applied science (sometimes considered as practical or normative science), its essence consists in formulating rules of how deductive inferences should be performed in order to fulfil their cognitive purposes. By contrast, logica docens has descriptive ambitions. It aims at a theoretical description of the world of logic, whatever this reality seems to be, ontological, epistemic or any other. Fortunately, my further analysis does not depend on the controversy between Platonism, conceptualism and nominalism.

Other important ideas for characterization of logic are expressed by such names as characteristica universalis (Leibniz), calculus rationicator (also Leibniz) or logica magna (systems of Frege, Russell and Whitehead as attempts to reconstruct the entire mathematics in one conceptual scheme). The next question important for the problem of the universality of logic consists in the plurality of logics. The variety of logics is considerable. One distinction is between the first-order (elementary) logic and higher-order systems. Furthermore, we have classical logic and several non-classical logics (many-valued, intuitionistic, paraconsistent, fuzzy, modal, non-monotonic, etc.). The problem of how they are mutually related is fairly complicated, 
because some logics are extensions of others (for instance, modal classical logic enlarges the classical system), but, on the other hand, we encounter the case of rivalry between particular logics and their extensions (for instance, classical and intutionistic logic, and consequently, classical modal logic and intuitionistic modal logic). Thus, we have differences, exclusions and overlapping even in a tentative map of contemporary logic. As far as the issue concerns logical rivalry, a word of caution is in order, because, for example, intuitionistic logic can be viewed as a competitor for classical one, but, from another standpoint, the former is a sublogic of the latter. The final distinction to be mentioned concerns logic with finite formulas and infinitary logic. In order to simplify my further considerations, I take first-order logic with identity (FOL) as the main system. Remarks on other logics will be marginal and restricted to some selected questions.

If we take FOL as a paradigm, three understanding of the universality property of logic might be discriminated:

(A) Logic is universal, if it is universally applicable;

(B) Logic is universal, if it is topic-neutral;

(C) Logic is universal, if its principles are universally true.

According to (A), every science as well as the commonsensical knowledge assume and apply logical rules, consciously or not. The view (B) denies that logic privileges any concrete subject. The point (C) claims that logical theorems are true in all circumstances, situations, states of the world, etc..

The statements (A)-(C) can be made precise by appealing to metalogical concepts and theorems. Let us take $C n$ as a primitive idea. Its axiomatization (I restrict it to propositional part of FOL with implication as the sole logical constant) is as follows:

(C1) $\emptyset \leq \mathbf{L} \leq \aleph_{0}$

(C2) $X \subseteq C n X$;

(C3) $X \subseteq Y \rightarrow C n X \subseteq C n Y$;

(C4) $C n C n X=C n X$;

(C5) $A \in C n X \rightarrow \exists Y \subseteq X \wedge Y \in \mathbf{F I N} \wedge(A \in C n Y)$;

(C6) $(A \rightarrow B) \in C n X \rightarrow B \in C n(X \cup\{A\})$;

The set (C1)-(C6) can be divided into two essentially separate groups. The first group includes (C1)-(C5) as general axioms for $C n$. (C1) says that the cardinality of a language $\mathbf{L}$ (understood as a set of sentences) 
is at most denumerably infinite, (C2) - that any set is a subset of the set of its consequences, (C3) establishes the monotonicity of $\mathrm{Cn},(\mathrm{C} 4)$ its idempotency, (C5) states the finiteness condition, which means that if something belongs to $C n X$, it belongs to the set of consequences of a finite subset of X. In other words, every inference is finitary, that is, performable on the base of a finite set of premises, which are, according to the character of syntactic rules, finitely long. (C1)-(C5) do not provide any logic in its usual sense, because they generate no rules of inference. The logical machinery is encapsulated by $(\mathrm{C} 6)$, that is, the deduction theorem. It define the connective $\rightarrow$, that is, implication.

Assume that $A, B$ are sentential formulas of $\mathbf{L}$ and $B \in C n\{A\}$ (in other symbolic vocabulary, this assumption can be written as the formula $A \vdash B$ saying that $B$ is provable from $A$ ). By (C6), we immediately obtain that $A \rightarrow B \in C n \emptyset$ (or $\emptyset \vdash A \rightarrow B$ ). Moreover, we stipulate that

(1) $A \in C n \emptyset$ if and only if $A \in \mathbf{L O G}$ ( $A$ is a logical theorem), and, generalize (1) to

(2) $\mathbf{L O G}=C n \emptyset$.

The last equality can be easily generalized for the entire FOL, that is, to the system with quantifiers and identity, provided that $\mathbf{L}$ consists of sentences (closed formulas). This condition is required by the deduction theorem. We can adopt (2) as the definition of logica docens, which says that logic consists from sentences deducible from the empty set of premises.

The definition (2) looks as strange at the first sight. In fact, one might ask "How is it possible to deduce something from the empty set?" or "How is logical creation ex nihilo possible?". We can think about this in the following way. Assume that LOG is axiomatized. Let the formula C denotes the conjunction of axioms and $\mathbf{t}$ be a theorem proved from $\mathbf{C}$. So the formula $\mathbf{C} \rightarrow \mathbf{t}$ is also a theorem. Add the new axiom $\mathbf{C} \in C n \emptyset$. By detachment, we obtain that $\mathbf{t} \in C n \emptyset$, as well. In order to justify the new axiom, one could eventually say that axioms are consequences of the empty set, because their acceptance does not logically depend on any premises (I do not consider pragmatic reasons, for instance, the deductive strength). In other words and according to the definition of proof as a sequence of formulas, writing an axiom constitutes its proof, being a one-term sequence in this case. On the other hand, if $A$ is a theorem of logic deducible from axioms, its proof has always a non-empty set of premises. Thus, if we use 
(1) and (2) as defining what logic is, we tacitly assume that logical axioms have a property, which is transferred from them to their consequences.

The above definition of logic is purely syntactic, because $C n$ belong to the vocabulary of syntax. The next step consists in employing semantics, which motivates

(3) $A \in \mathbf{L O G}$ if and only if for every model $\mathbf{M}, A$ is true in $\mathbf{M}$.

This definition describes logic as consisting of laws true in every model (domain, possible world, interpretation, etc), that is, $A$ is valid. Now, (3) is related to the weak completeness theorem:

(4) $A \in C n \emptyset$ if and only if $A$ is valid (other formulation, $\vdash A$ iff $\models A$ ). If we combine (3) and (4), validity, being a logical theorem and being a consequence of the empty set of premises, are coextensive. In other words, logic as defined by $\vdash$ (logic in the syntactic sense) and logic as generated by $\models$ fully coincide. Two other definitions of logic are equivalent to (2) (assuming (3) and (4)), namely (a system is a set of sentences closed by $C n$ ):

(5) $A \in \mathbf{L O G}$ if and only if $\neg A$ is inconsistent.

(6) LOG is the only non-empty product of all consistent deductive systems (theories).

Since both are intuitively plausible, the same concerns (2).

The settings (1)-(6) essentially contribute to the characterizations of the universality property captured by (A)-(C). In particular, (3) corresponds to universality as being universally true (valid) as well as to topicneutrality. The first correspondence is obvious, but the second one has its justification in the fact that if no model is priviledged, logic is topically neutral. This property is additionally supported by the theorem that logical tautologies do not distinguish any extralogical content (see also below). Furthermore, if logic belongs to every theory, it is universally applicable. Finally, we can prove that logica docens and logica utens are equivalent. Think about the latter as a system of rules having the form

(*) $A_{1}, \ldots, A_{n-1}, A_{n} \vdash A$.

By $(\mathrm{C} 6),(*)$ becomes the formula

$$
(* *) A_{1} \rightarrow\left(\ldots\left(A_{n-1} \rightarrow A_{n}\right) \ldots\right),
$$

which is valid. This assertion follows the earlier remark that the results of functioning of the operators $\vdash$ and $\models$ coincide. 
I return to the problem of the universality of logics. Since we have various logical systems, we must pose the question which logic possesses this property in the sense established above? The answer that

$(\bullet)$ FOL is a natural candidate, because the weak completeness theorem holds for it.

looks plausible. The Lindstrm theorem (every logic, which satisfies the compactness theorem and the downward Löwenheim-Skolem theorem is equivalent to FOL; compactness can be replaced by completeness, but it is a stronger assumption) provides an additional evidence for the above conclusion. In particular, the Löwenheim-Skolem property (possessing a denumerable model, if having a model of any infinite cardinality) marks that FOL does not distinguish any extralogical content. In other words, having non-standard models (in this case, of different infinite cardinalities) is not at odds with the universality property of logic.

Yet "What about other logics?" - one can ask, because the completeness theorem holds not only for FOL. Well, this question has several aspects. A brief report is as follows. Firstly, model theory for FOL does not assume any special conditions for its models. By contrast, other logics, higher-order, intutionistic, modal, many-valued, paraconsistent, fuzzy, non-monotonic etc. are associated with special structures. For instance, semantics for modal logics imposes special constraints for the accessibility relation (symmetry, transitivity, etc.). Thus, this circumstance does not devastate the special status of FOL in the family of systems called pretending to be a logic. Secondly, FOL is based on some additional conditions. Its language is at most denumerable, compositional and consists of finitely many formulas, generalized quantifiers are excluded, identity is a logical constant or empty individual constants are not admitted. Moreover, $\mathrm{Cn}$ for FOL satisfies (C5), that is, it is finite. We have no other justification for these properties than appealing to intuitions or/and pragmatic factors, like familiarity, simplicity, logical naturality, etc.. In particular, we can say that there is no way to operate by using non-denumerable languages, infinitely long formulas as well as to infer something from infinite sets of premises. On the other hand, one might reply that we are able to describe such linguistic monsters and admit the $\omega$-rule. Assuming compositionality is an obvious simplification because several intensional logics or Hintikka's independence-friendly logic have non-compositional languages. Consequently, various aspects of FOL are related to more or less con- 
ventional settings. Thirdly, any analysis of logic requires formal devices employed in the metalanguage. Are they universal or not? In particular, we use very elementary set theoretical devices in presenting concrete logics, for instance, concepts expressed by such sings as $\in$, $\subseteq$, etc. We also use logical constants in the metatheory and their meaning is the same as in L. Philosophically speaking, this procedures can be accused of involving a hermeneutical circle. However, this remark concerns any logic, not only FOL. In particular, there is a question whether reasoning about non-classical logics can be adopted in the framework of a given logic or requires FOL. The case of intuitionistic logic is perhaps the most typical one. Is it possible to prove the completeness theorem for this logic using intuitionistically acceptable principles? The positive answer to this question is dubious, at least until now (see [18]).

Although as the above discussion suggests, some issues concerning FOL are still (and probably will be) debatable, the thesis (the first-order thesis)

(FOT) FOL is the only logic having the universality property in the sense of $(\mathrm{A})-(\mathrm{C})$ and is the logic.

has a support. On the other hand, this thesis has been strongly attacked in the last thirty years (see [1]). The principal argument says that FOL is too poor for analysing mathematical structures and mathematical concepts. A typical example is that this logic is too weak to define the concept having no sufficient expressive power (due to the compactness theorem) to capture the notion of being a finite set (this limitation is). On the other hand, the criticism does not claim that FOL should be rejected, but points out that it should be somehow extended (see [4], [8], for general surveys). Such extensions are labelled as extended model theory, theory, abstract logic, abstract model theory, model-theoretic logic (all names are related to views expressed in [1]), algebraic logic (see [10], [7]), protoalgebraic logic (see [5]), categorical logic (see [9], [12], [13]) or universal logic (see [2]).

The first remark about the criticism of FOT should point out that the assumed concept of universality is different from the one coded by $(\mathrm{A})-(\mathrm{C})$. It is captured by

(D) Logic is universal if it has a great expressive power.

This means that universality of logic is measured (at least, intuitively) by its ability to cover (define, explicate, etc.) various mathematical concepts. It also means that the concept of logic becomes changed. To remind, logic 
was hitherto understood as LOG (a system of theorems and/or rules) together with metalogic. The point $(\mathrm{D})$ is rather related to understanding of logic as the pair $<\mathbf{L}, \models^{\mathbf{M}}>$, where the sign $\models^{\mathbf{M}}$ expresses the concept of being satisfied in a model $\mathbf{M}$. Briefly, logic is a language together with a class of models (structures). In this setting, FOL is a first-order language with $C n$ (or $\vdash$ ). In general, $\langle\mathbf{L}, \vdash\rangle$ depicts a formal apparatus used in $\mathbf{L}$ of a given kind related to specific features of this language (its order, length of formulas, types of quantifiers, cardinalities of sets of premises, etc.). Consequently, only axioms for (C2)-(C4) are accepted for $\mathrm{Cn}((\mathrm{C} 3)$ is dropped in the case of non-monotonic logic). If we use logic in the sense $<\mathbf{L}^{1}, \models{ }^{\mathbf{M}}>$, where $\mathbf{L}^{1}$ is a first-order language, the first-order model theory is qualified as too poor for offering resources for mathematical investigations.

A digression on further understandings of universality. At least five other senses of universality are of interest for the philosophy of logic. Firstly, we have the meaning presented by the phrase "universal grammar". Universal grammar looks for universal categories present in all languages. Secondly, there is universal algebra, that is, investigations of various algebraic systems, like groups, fields, rings, etc. as structures defined by the properties of algebraic operations, for instance associativity, transitivity, etc. Thirdly, the category theorists speak about universal constructions. Very roughly, whereas the traditional strategy consisted in defining objects, for instance, natural numbers, the categorical way tries to define a property which universally holds with respect to a given morphism. Fourthly, universality can be viewed as unrestricted quantification. Karl Popper considered the universality in this sense as particularly valuable in the case of empirical theories. Fifthly, $a$ is more universal than $b$ if $b$ is a special case of $a$.

All understandings listed in the above digression can be applied for logic. Firstly, logic looks for logical invariant in all languages. Logical constants can be viewed from this point of view, even if they are only approximations of what occurs in natural language. Secondly, according to some logicians (see [3], p. 1), model theory is logic plus universal algebra. Moreover, the idea of universal logic is strongly motivated by universal algebra. More specifically, universal logic generalizes particular systems of logic. Thirdly, categorical logic tries to define universal constructions in the sense of category theory (see below). Fourthly, quantification over models can be unrestricted as in the case of FOL or restricted as applied to other logics. 
Yet one additional remark is in order here. The problem of unrestricted quantifications is frequently discussed (see papers in [14]) as the question of possibility of a discourse with all possible objects. Clearly, various metamathematical results show that several limitations have to be introduced while speaking about universes in order to avoid contradictions. Fifthly, logic often speaks about general and special cases. For instance, we say that an individual constant is a zeroary function symbol (it has no arguments). This means that individual constants are special cases of function symbols. Yet one should be careful in applying this mode of speaking to logic. Typically, we say that classical mechanics is a special case of the relativity theory, and that the latter is more universal then the former. However, take many-valued logic and three-valued logic. According to my earlier explanations, FOL is universal, because it consists of theorems true in all models. On the other hand, two-valued logic can be regarded as a special case of many-valued logic, provided that the set of logical values is restricted to $\{$ VERUM, FALSUM $\}$. Similarly and according to (6), if FOL is a part of every deductive system, then if $\mathbf{S}$ is a special case of every system that has extralogical axioms. These observations suggest that logical universality differs from other kinds of universality, at least in some cases.

Perhaps categorical logic, more and more popular, in the last few decades is a good illustration of (D). This way of looking at logic can be considered as a continuation of algebraic logic. It started with Boole and his observation that operations between sentences are parallel to relations holding between sets. Cylindric algebra was applied to algebraization of quantifiers (see [11]). The next step consisted in introducing algebraic (also other, for instance, topological and categorical) methods in metamathematics (see [6], [15]). The main idea of categorical logic consists in shaping logic as the pair:

(7) < Formulas as objects, Morphisms (Arrows) as rules of inferences $>$. Consequently, logic becomes a mathematical (even algebraic) item, which, in this case, is investigated by methods of category theory. The main work consists in defining such concepts as soundness, completeness, consistency, etc. As far as I know the present shape of categorical logic is a mixture of the traditional approach and new ideas since not all metalogical concepts and properties got defined in category theory. Moreover, informal classical logical inferential rules are employed in proofs within category theory. 
Interesting results achieved in categorical logic concerns intutionism. Consider the category Top (toposes). It was proved that the logic of toposes is intutionistic. This result is perhaps surprising, because the definition of a topos does not suggest that the properties of such objects generate intuitionistic logic. Yet if one notes that topoi are a generalization of topological spaces, this connection could be expected due to the well-known links of topology with intuitionistic logic, mostly established by Alfred Tarski and his students (see [16], Chapter XVII). Another example is provided (see [16], p. 352) by the relation between the usual algebra of set (governed by classical logic) and the calculus of systems (subjected to intutionistic logic). Category theory leads to the distinction between internal and external logic of a given category. For instance, whereas internal logic of Top is intutionistic, the external one - classical (or at least weaker). If we consider Set (the category of sets), its logic is classical, internally and externally. Several traditional foundational problems return in category theory. How to think about Cat, intended as the category of all categories? The question is, of course, related to set-theoretical paradoxes. We have first-order categorical logic and its higher-order version. This distinction immediately leads to the question concerning the limits of the former as possessing too restricted expressive power. Although the issue is perhaps not so hot as in the case of controversy over FOT, because we have to do more with a part of mathematics than with logic in its traditional sense, both discussions are obviously related. To sum up (perhaps tentatively and modestly), many foundational problems debated in more traditional foundational studies, returns when the conceptual apparatus of category theory is used. Although it would be an exaggeration to say that category theory solves difficulties, it certainly opens new mathematical perspectives.

It seems that both understanding of universality, namely (A)-(C) as well as (D) should be taken into account with no deliberation on which is more important. In fact, they are reversely proportional, because more expressive power limits the class of models. It is exactly as in traditional case: greater intension - smaller extension and reversely. Logical validity is (and should be) maximal in a somehow maximal sense. On the other hand, expressive power being correlated with truth is not measurable in all models. Hence, there is no compromise between the property of topical neutrality and categoricity. FOL cannot be categorical (in the traditional understanding) and it is not its bad feature. In particular, the Lwenhein- 
Skolem puzzle is unjustly treated as a paradox. If we say that logic does not distinguish extralogical contents and we expect this property, this puzzle is very natural, because logic as such does not provide resources for assessing cardinalities of models. Thus, we should discern not only two kinds of universality, but even two kinds of logic. Formally speaking, $\left\langle\mathbf{L}^{1}, \vdash\right\rangle$ and $<\mathbf{L}^{1}, \models>$ coincide, due to the strong completeness theorem, but, on the other hand, $\left\langle\mathbf{L}^{n}, \vdash>\right.$ and $\left\langle\mathbf{L}_{n}, \models^{\mathbf{M}}\right\rangle$, not always, but if they do, some special assumptions must to be adopted. There are ways of using, for instance, categorical logic. Firstly, we can consider logic as an object investigated in category theory, and, secondly, to apply categorical apparatus in metalogic (metamathematics) traditionally conceived. This raises the question of how strong devices used in the metalogical sense should be. For mathematicians, this problem may be of some interest, due to their professional interests. Philosophers' situation is different. As I have mentioned earlier, they must confront the problem of circularity of analysis of logic, which calls for the weakest tools, perhaps like in the case of reverse mathematics. Anyway, my philosophical taste favours FOT and putting (D) on the level of metatheory.

\section{References}

[1] J. Barwise, Model-Theoretic Logics: Background and Aims, [in:] J. Barwise, S. Feferman, (eds.), Model-Theoretic Logics, Springer, Berlin (1985), pp. 3-23.

[2] J.-Y. Béziau, A. Costa-Leite (eds.), Perspective on Universal Logic, Polimetrica, Monza (2007).

[3] C. C. Chang, H. J. Keisler, Model Theory North-Holland, Amsterdam (1977).

[4] J. P. Cleave, A Study of Logics, Clarendon Press, Oxford (1991).

[5] J. Czelakowski, Protoalgebraic Logic, Kluwer, Dordrecht (2001).

[6] R. Diaconescu, Institution-Independent Model Theory, Birkhäuser, Basel (2008).

[7] J. M. Font, Abstract Algebraic Logic An Introductory Textbook, College Publications, London (2016).

[8] D. Gabbay, (ed.), What is a Logical System?, Clarendon Press, Oxford (1994). 
[9] R. Goldblatt, Topoi. The Categorical Analysis of Logic, NorthHolland, Amsterdam (1979).

[10] P. Halmos, S. Givant, Logic as Algebra, Mathematical Association of America, New York (1998).

[11] A. Heyting, D. Monk, A. Tarski, Cylindric Algebras, Part I, NorthHolland, Amsterdam (1971).

[12] J. Lambek, P. J. Scott, Introduction to Higher Order Categorical Logic, Cambridge University Press, Cambridge (1986).

[13] M. Makkai, G. E. Reyes, First Order Categorical Logic, Springer, Berlin (1977).

[14] A. Rayo, G. Uzgquiano (ed.), Absolute Generality, Clarendon Press, Oxford (2006).

[15] H. Rasiowa, R. Sikorski, The Mathematics of Metamathematics, PWN, Warszawa (1970).

[16] A. Tarski, Logic, Semantics, Metamathematics, Clarendon Press, Oxford (1956).

[17] J. Woleński, First-Order Logic: (Philosophical) Pro and Contra, [in:] V. Hendricks, F. Neuhaus, S. A. Pedersen, U. Scheffler, H. Wansing

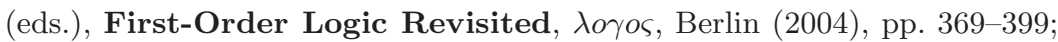
reprinted [in:] J. Woleński, Essays on Logic and Its Applications in Philosophy, Peter Lang, Frankfurt am Main (2011), pp. 61-80.

[18] J. Woleński, Constructivism and Metamathematics, [in:] A. Koslow, A. Buchsbaum (eds.), The Road to Universal Logic. Festschrift for 50th Birthday of Jean-Yves Béziau. Vol I, Birkhäuser, Basel (2015), pp. $513-520$.

[19] J. Woleński, Normativity of Logic, [in:] J. Stelmach, B. Brożek, Ł. Kwiatek (eds.), The Normative Mind, Copernicus Center, Kraków (2016), pp. 169-195.

University of Information, Technology and Management

Rzeszów, Poland

e-mail: jan.wolenski@uj.edu.pl 